This is the Accepted Manuscript of an article published by Taylor \& Francis in "Expert Review of Molecular Diagnostics" on $26 / 07 / 2018$.

Giorgio Gallinella (2018) The clinical use of parvovirus B19 assays: recent advances, Expert Review of Molecular Diagnostics, 18:9, 821-832, DOI: 10.1080/14737159.2018.1503537

Available online:

https://doi.org/10.1080/14737159.2018.1503537 


\title{
The clinical use of parvovirus B19 Assays: recent advances
}

\author{
Department of Pharmacy and Biotechnology \\ University of Bologna, Bologna, Italy
}

Running Title: The clinical use of parvovirus assays

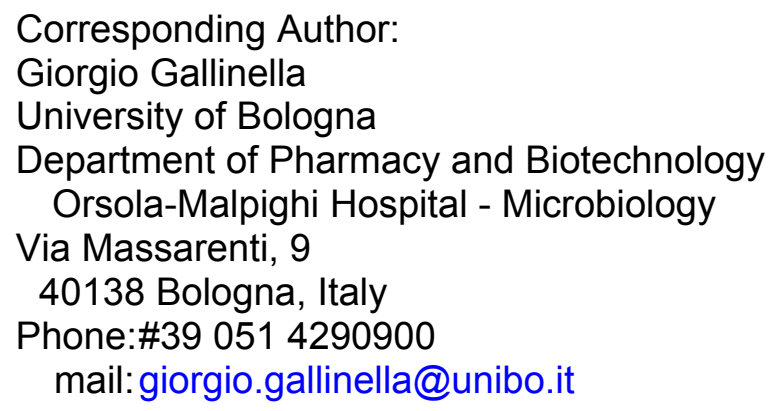




\begin{abstract}
Introduction: Parvovirus B19 (B19V), a single-stranded DNA virus in the family Parvoviridae, is a human pathogenic virus, characterized by a selective but not exclusive tropism for erythroid progenitor cells. Widely diffuse, it is responsible for an ample range of clinical manifestations, whose characteristics and outcomes depend on the interplay between the viral properties and the physiological and immune status of the infected individuals. The complexity of virus-host relationship and the diversity of the clinical course of infection pose a diagnostic challenge that may require non trivial solutions.
\end{abstract}

Areas covered: The review includes an updated description of the course of B19V infection in its complexity and diversity of pathogenetic mechanisms, discusses the consequent requirements for different and appropriated diagnostic approaches, presents the main diagnostic techniques, more recent technical advancements, and their application to the diverse clinical situations.

Expert commentary: The complex scenario of the infectious process and the diversity in possible pathogenetic mechanisms make necessary a multi-parametric approach for an accurate and informative laboratory diagnosis of B19V infection, combining as much as possible the molecular detection of viral components, mainly viral DNA, to commonly followed immunological detection of virus-specific antibodies and a critical assessment of laboratory findings.

\title{
Keywords
}

antibody detection; molecular diagnostic; Parvovirus B19; qPCR assays; virus-host relationship; 


\section{Introduction}

\subsection{Taxonomy}

The family Parvoviridae includes viruses with a single-stranded DNA genome, encapsidated in a icosahedral protein capsid, about 22-26 nm in diameter. Replication occurs in the nucleus of infected cells and is highly dependent on cellular environment, so that a productive cycle is usually achieved only in actively dividing cells, or in some cases when supported by complementation from helper viruses. The subfamily Parvovirinae includes viruses infecting vertebrate hosts, within it the most recent taxonomical revision distinguishes eight viral genera, and within each genus individual virus species that collect the viral isolates normally recognized in clinical or laboratory settings. In the genus Erythroparvovirus, the species Primate erythroparvovirus 1 includes the human parvovirus B19 (B19V), and a further subdivision is introduced in three distinct B19V genotypes, that differ in their genome sequence by about $10 \%$, while constituting a single serotype and showing similar biological properties [1].

Viruses adapted to the human host are found in the genera Dependoparvovirus (Adeno-Associated Viruses, AAV), Erythroparvovirus (B19V), Bocaparvovirus (HBoV1-4), Tetraparvovirus (PARV4). While AAV viruses are considered non-pathogenic and have been exploited as transduction viral vectors, the others possess a pathogenic potential that prompts for the development of diagnostic molecular testing in a clinical setting. B19V is a widely circulating virus implicated in a complex relationship with the host, and can be considered the most relevant human pathogenic virus in the family, posing the necessity of diagnostic awareness and appropriateness. The aim of this review is to present the more recent contributions to our knowledge on the course of virus infection, on its pathogenetic mechanisms, and on the appropriate molecular diagnostic methods.

\subsection{The virus and its biology}

B19V shares structural features with other viruses in the family (for more extended reviews, see refs $[2,3])$. The genome is a linear ssDNA molecule of $5.6 \mathrm{~kb}$ in length, strands of either polarity are separately encapsidated at the same frequency and are functionally equivalent. The genome organization is composed of a unique internal region, containing all the coding sequences, flanked by repeated, inverted terminal regions that serve as origins of replication. The unique internal region encodes for three major proteins, the non-structural protein NS in the left side, and the two colinear capsid proteins, VP1 and VP2, in the right side, and for additional minor non-structural proteins. The capsid, composed of 5-10\% VP1 and 90-95\% VP2 proteins, forms an icosahedral structure in $T=1$ arrangement, about $25 \mathrm{~nm}$ in diameter. VP proteins produced in heterologous expression systems can self-assemble into viral-like particles (VLPs) that are structurally analogous to native virions and are used in diagnostic immunoassays, as well as for a candidate vaccine antigen (Figure 1). 
B19V shows a selective tropism for erythroid progenitor cells in the bone marrow, due to the presence of specific receptors, such as the glycolipid globoside and a specific receptor binding the VP1 unique N-terminal domain, and to functional internalization processes [4, 5]. In a permissive cellular environment, a coordinated series of macromolecular syntheses occurs [6-8]. From the parental single-stranded template, cellular DNA repair synthesis generates a double stranded DNA template, then a first phase transcription mainly produces mRNAs coding for the NS protein, followed by rolling hairpin replication of the genome and extended second phase transcription, mainly producing mRNAs coding for structural VP proteins. Accumulation of VP proteins eventually leads to the assembly of capsids, encapsidation of progeny single-stranded genomes and release of virions from infected cells. The permissive environment is restricted to cells in the erythroid lineage at differentiation stages ranging from CFU-E to erythroblasts [7], and is critically dependent on Erythropoietin stimulation and hypoxic conditions [6, 8], through a signaling cascade eventually leading to formation of a functional replicative complex involving the viral NS and cellular proteins [9]. In productively infected cells, the virus exerts a complex series of effects, including arrest of the cell cycle and induction of apoptosis [10], thus causing a temporary block in erythropoiesis that can manifest as a transient or persistent erythroid aplasia. The virus can infect other different cellular types in diverse tissues, including endothelial, stromal, or synovial cells. However, cellular environments other than erythroid progenitor cells are normally non-permissive to viral replication, so in these the presence and persistence of the viral genome may not be associated with its replication, transcription or protein synthesis. In non-erythroid tissues, although a productive viral replication that may contribute directly to pathological processes can be sporadically documented, infection is usually abortive and the virus is supposed to exert its pathological potential by indirect mechanisms, such as the induction of inflammatory or autoimmune processes (extensively reviewed in [11] and critically discussed in [12]).

\section{Course of infection}

B19V is widely and worldwide diffuse, and infection can be associated with an ample range of pathologies and clinical manifestations, from the asymptomatic or mild to the severe and in some cases life-threatening, whose characteristics and outcomes depend on the interplay between the viral properties and the physiological and immune status of the infected individuals (for more extended reviews, $[2,3])$. The complexity of virus-host relationship and the diversity of the clinical course of infection pose a diagnostic challenge that may require non-trivial solutions.

\subsection{Transmission}

In most epidemiological settings, considering the presence of specific lgG as the marker of occurred infection, the highest force of infection occurs before age of 20 , reaching a prevalence of about $60 \%$ in the population aged $20-30$ years. Infection can occur until elder ages, reaching 
maximal prevalence values higher than $80 \%$, and data fitting epidemiological models indicate the best scenarios allowing for waning immunity at an age-specific rate over the maintenance of lifelong immunity, assuming that the transmission rates are directly proportional to the contact rates. The main route of transmission of the virus is through the respiratory system, estimated overall $R_{0}$ values are $\sim 2.4$, and duration of contacts is the main determinant of transmission, so that prolonged contacts as in the household and community settings are especially effective in transmission. In temperate climate countries, circulation of the virus is higher in the Spring/early Summer months, and epidemic cycles are reported to occur. For sophisticated epidemiological models and related data, see [13-15]. The virus can be transmitted from mother to fetus posing a risk of fetal damage that should prompt for an antenatal assessment of risk of fetal infections and diagnostic attention towards the development of intrauterine infections (see [16-18] for recent reviews). Finally, due to a viremic phase with high viral load, there is a risk of iatrogenic transmission of the virus via blood and blood-derived products, implying blood and blood product safety issues (see $[19,20]$ for recent reviews).

\subsection{Infection - Early Events}

Following contact and a primary viremic phase, normally unsuspected and undetected, the virus gains access to the bone marrow and infects erythroid progenitor cells, achieving a productive infection and exerting cytotoxic effects [21]. In this phase, upon cytological examination, the bone marrow shows erythroid aplasia and the presence of characteristic giant erythroblasts (for sample images, [22, 23]). The pathogenic effects on bone marrow are derived from the ability of the virus to induce cell-cycle arrest, block of erythroid differentiation, and eventually apoptosis of susceptible and infected cells. At this stage, the clinical impact on the host reflects the depression of bone marrow activity, linked to the volume and turnover rate of the erythroid compartment, and from the ability of immune system to mount an effective specific response.

In individuals with physiological erythropoiesis and normal immune system, infection is limited in extent and temporal frame, and is controlled by the development of a specific neutralizing immune response. Levels of hemoglobin decrease only marginally and infection is usually asymptomatic from the hematological perspective. Production of antibodies with neutralizing activity (IgM followed by $\operatorname{lgG}$ ) contributes to the progressive clearance of infection with constantly decreasing viral load levels. When preexisting alterations in the erythropoiesis process, either due to genetic defects or stressed physiological conditions, or defects in the immune response, alter the balance between viral replication and cellular turnover, infection can manifest as pure red-cell aplasia (PRCA) and anemia [24]. In situations where the number of erythroid progenitors and their replication rate is expanded because of a reduced lifespan of erythrocytes, or increased need, infection can lead to an acute episode of profound anemia, presenting as classical aplastic crisis. In situations where the immune system lacks the capacity to control, neutralize, and clear viral infection, the infection may become persistent, depression of erythropoiesis can manifest with chronic anemia of different 
grades, but otherwise erythropoiesis can be compensated and infection clinically unapparent. In rare instances, the virus has been linked to bone marrow necrosis [25], and more in general to a wide variety of blood diseases and cytopenias other blood cell lineages than the erythroid [21], by mechanism that still require investigation.

\subsection{Infection - Late Events}

Bone marrow supports a productive infection and release of progeny virus into the blood, leading to a secondary viremia characterized by high viral load levels (up to $10^{12}$ virus $/ \mathrm{mL}$ ) in the acute phase, further leading to a systemic distribution of the virus and preluding to possible late clinical manifestations of infection. In this respect, the two classical late manifestations of B19V infection are erythema infectiosum typical of children, and arthropathies typical of adult patients, this latter with a tendency to chronicity, but the virus has been implicated, more or less convincingly, as etiological agent in a wide range of pathological processes. In particular, in recent years, B19V has been recognized as a main cardiotropic virus, and its implication in the development of acute or chronic myocardiopathies has been the subject of intense debate (recently summarized in [26]). Also, the involvement of $\mathrm{B} 19 \mathrm{~V}$ in the development of autoimmune disorders has been the subject of intense investigation [27], and possible pathogenetic mechanisms involving the formation of apoptotic bodies induced by NS protein expression have been proposed [28].

In most cases, late clinical manifestations occur in the presence of a specific immune response, so that immune-mediated inflammatory processes are assumed. However, it is in this phase that different non-erythroid cell types, including endothelial, stromal or synovial cells, can also be infected [11, 12]. While these cell types are normally non permissive to viral replication, markers of viral activity have in some cases been precisely localized within cells, such as endothelial or synovial cells, and causally linked to pathological processes by a direct viral-induced pathogenetic mechanism. Over the years, the spectrum of clinical manifestations associated to B19V infection has been constantly increasing to involve almost all organs and tissues, and descriptions of clinical presentations have progressively stressed atypical aspects. Sound methodologies and strict diagnostic criteria should always be adopted to link B19V infection to atypical pathological processes.

\subsection{Virus Persistence}

Following primary infection, B19V, or at least B19V DNA, can be detected in peripheral blood for prolonged periods, and can then persist in a wide range of tissues, probably lifelong [11, 12], raising questions about the actual relevance and pathogenetic implications of these findings. In peripheral blood, viremia measured as the detection of viral DNA is considered a marker of active infection. However, low levels of viral DNA can be detected for extended period of times following a documented past infection [29,30], and be a rather common finding in the normal population as well [31]. Presence of viral DNA in blood has been documented in subjects with congenital or 
acquired immunodeficiency, in patients receiving chemotherapy for hematological or solid malignancies [32, 33] and in the course of immunosuppressive treatments in bone marrow [34, 35] or solid organ transplant recipients [36-39]. Presence of viral DNA is interpreted as indicating the occurrence of persistent infections, although not necessarily associated with underlying pathogenetic mechanisms and with clinically relevant situations. Recently, it has been demonstrated that an analytical differentiation can be introduced between the detection of viral DNA within virions, thus related to an active productive infection, and circulating free viral DNA, possibly released from sites of persistence in the tissues, in a concept similar to that of liquid biopsy [40]. The clinically utility of such differentiation has probably just begun to be appreciated [41].

Concerning persistence in tissues, a comprehensive review of available published information indicates that the average overall frequency of samples positive for B19V DNA in all tissues, including bone marrow, lymphoid tissues, liver, heart, synovia, skin, testis, thyroid, and brain is as high as $44.8 \%$ [11]. The virus can be detected in bone marrow, the primary target organ, not only in cases of active infections with constant low-level viremia, but also in $\sim 20 \%$ of biopsies from subjects without any evidence of active viral replication, and can be detected also in lymphoid tissue, including spleen, lymph nodes, and tonsils [11]. Viral DNA can be detected in liver, and in the heart the common presence of B19V DNA has raised attention on its potential role in the development of cardiomyopathies [42-44]. Viral DNA is commonly found in synovial tissues, thus with little informative content when trying to relate its presence to the development of rheumatologic diseases [27], and skin, with similar implication with respect to dermatological issues $[45,46]$. In all these instances, large-scale surveys have demonstrated the persistence of viral DNA in substantial fractions of sample populations, independently of clinical associations, so its mere detection cannot be assumed as a diagnostic criteria suggestive of a virus-driven pathological process. Additional parameters, such as the demonstration of expression of viral mRNAs and proteins, need to be assessed to postulate B19V as the pathogenetic agent in observed concurrent alterations in host cells and tissues and related clinical manifestations.

On the whole, the initial idea of a virus capable of acute infections but rapidly cleared from the organism has given place to the concept of a virus able to establish long-term relationship with human hosts, and the current assumption is that persistence of viral DNA in tissues can be the normal outcome of infections, making B19V a relevant part of the human virome in its expanding concept [47-49]. Strikingly, viral DNA detected in tissues can provide an archive for ancient genomic variants that are not currently circulating, bearing witness to an active evolutionary process involving B19V and leading to its actual differentiation in the three recognized genotypes $[50,51]$. What is observed in the population is a stratification of archived variants in the different age groups, from ancient and extinct to recent and circulating variants. Whether this persistence might be due to silent maintenance of viral genomes as episomes, to integration of viral DNA in the 
host genome as recently observed in erythroid cells in vitro [52], or be related to periodical reactivation from latent reservoirs leading to productive infections, is still a matter of investigation [12]. Until a better understanding of the characteristics of this long-term relationship is obtained, and pathogenetic mechanisms are better understood, so the relevance of diagnostic markers will continue to be a matter of debate, requiring careful assessment and interpretation for clinical utility.

\subsection{B19V as a cardiotropic virus}

The interpretation of the presence of viral DNA in tissues as a clue to the development of pathologies is nowhere such controversial as for its detection in heart tissues. As already stated, in recent years, B19V has gained interest as a cardiotropic virus, being detected at ever increasing frequencies in endomyocardial biopsies, replacing other cardiotropic viruses as the most prevalent virus detected in the heart, and a marked cardiotropism should be mentioned as one of the main characteristics of $\mathrm{B} 19 \mathrm{~V}$. A recent extensive review summarizes the still partial and contradictory findings collected until now on the role of B19V in the development and clinical course of myocarditis and chronic dilated cardiomyopathy [26].

It is demonstrated that $\mathrm{B} 19 \mathrm{~V}$ can infect myocardial endothelial cells, thus a proposed (but not thoroughly validated) pathogenetic mechanism would involve in sequence a direct cytotoxic and proinflammatory activity exerted by viral NS and VP1 proteins on endothelial cells, then followed by recruitment and activation of inflammatory and immune cells leading to endothelial disfunction and CTL-mediated tissue damage, and finally to persistence of virus in tissues, possibly associated to chronic inflammation and cardiomyopathy. Such pathogenetic mechanism would require not only infection of endothelial cells, but also viral replication or at least expression of viral proteins, which is a testable hypothesis through quantitative determination of viral DNA per number of cells and detection of viral mRNAs and proteins in endomyocardial biopsies [26].

On these grounds, B19V has been directly involved as an etiologic agent in acute myocarditis both in pediatric [53] and adult populations [54]. On the other hand, the reported frequency of detection of B19V DNA in endomyocardial biopsies in sample populations, controls as well as patient groups, shows a wide variability, is usually higher in recent works that can probably use more sensitive detection methods, and as a consequence there is no consensus on the relevance of the presence of viral DNA either as a diagnostic or prognostic parameter in the development of cardiomyopathies, in particular dilated cardiomyopathy and ventricular dysfunction. A suggestion for involvement of B19V in coronary artery disease has also been reported [55]. In fact, a significant pathogenetic association has been hypothesized because of significant higher frequencies of B19V DNA detection in patient versus control groups, or of the presence of higher mean viral loads suggesting active viral replication. However, by using these same indicators, a lack of significant clinical association has also been recently advocated [56]. Reasons for these discrepancies are not readily evident, and may involve sample population composition, different 
selection of clinical parameters and evaluation endpoints, inherent variability in tissue sampling and differences in the molecular assessment methodologies. Investigation of markers of viral activity, such as the presence of viral transcripts or viral proteins, may better identify subset of patients where B19V acts a more direct pathogenetic role [57]. The rare occurrence of clinically relevant myocarditis and development of cardiomyopathies compared to the widespread diffusion of $\mathrm{B} 19 \mathrm{~V}$ infections underscores the relevance of coincident factors, that are presently ignored. A predisposing genetic background, viral coinfections (e.g., with HHV-6), the extent of vascular damage [58], the activation of the innate immune system, or the characteristics of the adaptive specific anti-B19V immunity, can all contribute critically in the development of B19V-related cardiomyopathies. Pertinent in vitro experimental models and standardization of analytical procedures will be required in future studies to obtain reliable information on the role of $\mathrm{B} 19 \mathrm{~V}$ in the development of acute or chronic cardiomyopathies.

\subsection{Fetal Infection}

A relevant property of $\mathrm{B} 19 \mathrm{~V}$ is its ability to cross the placental barrier and infect the fetus [16]. When in the fetal circulation, the virus can infect erythroid progenitor cells, in liver and/or bone marrow depending on the gestational age, and can be detected in erythroid cells circulating in the vessels of several tissues, in endothelial placental cells as well as in the amniotic fluid. The virus can induce a block in fetal erythropoiesis whose effect will depend on fetal developmental stage, the rate of expansion of fetal erythroid compartment and the maturity of the immune response. Infections occurring at earlier stages of pregnancy carry a higher risk of fetal death, up to about $10 \%$, while infections occurring in the central part of pregnancy more frequently lead to fetal hydrops. Hydrops may eventually cause fetal death, but frequently the fetus can recover without persistent developmental damage. In the third trimester the overall risk of fetal damage decreases to background values, although late intrauterine fetal death can occur [17, 59]. Conservative management of documented infections is advised, with an indication for improved survival following intrauterine transfusions in case of documented fetal anemia below a threshold level [60]. Newborns may show transient presence of virus at birth, only sporadically associated with neonatal anemia or anomalies [18]. Parvovirus B19 infection during pregnancy is not associated with increased morbidity or mortality in infancy and childhood [61], while consequences of fetal anemia on the long-term neurological development are hypothesized, but still under investigation [62]. In view of the fact that effective protocols are established for an appropriate management of intrauterine infections, it is rather contradictory that antenatal screening and generalized surveillance are not recommended [63, 64], although their introduction would prove useful $[65,66]$. As a consequence, diagnostic algorithms mostly focus on a diagnosis of suspected active infection in pregnancy and on confirmation of fetal infection. Usually antibody detection in maternal blood are sought as a first-level investigation, but in most instances it will be necessary to include a molecular detection of virus in maternal blood as well as on fetal specimens to achieve a definite 
diagnosis and guide clinical management $[67,68]$, including recommendation for intrauterine transfusion [69].

\section{Molecular detection assays}

\subsection{Specimens and Molecular Targets}

The low structural complexity of B19V restricts the range of molecular targets relevant for a direct diagnosis of infection mainly to viral DNA. Detection of viral DNA in serum/plasma from peripheral blood, other body fluids, fetal cord blood, or amniotic fluid, is an indication of productive viral replication and active infection. Due to the characteristics of the viral replication and the dynamics of the infectious process, it is critical for diagnostic methods to produce a reliable quantitative assessment of the viral load in order to obtain useful diagnostic information. Furthermore, recent indication is emerging for the analytical differentiation between the detection of viral DNA within virions, and circulating free viral DNA. Viral DNA can also be sought in cellular samples, such as bone marrow aspirates, solid tissue biopsies, fetal tissue or placenta. When investigating tissue samples, in order to differentiate active infections from silent persistence of virus, both quantitative evaluation of the genome copy number and detection of viral mRNAs can be considered appropriate. To this purpose, besides quantitative molecular amplification methods, viral nucleic acids may be detected by in situ hybridization, or viral proteins can be detected by immunological methods.

\section{2. qPCR Techniques}

Over the years, in the progress towards a rapid and accurate molecular diagnosis, a wide array of molecular hybridization and nucleic acid amplification techniques have been developed. Currently, quantitative and internally controlled real-time PCR techniques (qPCR) represent the standard analytical method for the molecular detection of B19V DNA. Two main requirements should be met: (i) the capability of detection of all genotypes of B19V, and (ii) a calibrated and standardized quantification of target. Both of these take advantage of international standards [70] and can be challenged by international proficiency panels.

B19V Genotypes. B19V as a species is subdivided into three genotypes, the prototype genotype 1 , and two variant genotypes 2 and 3 . At the nucleotide level, the diversity between genotype clusters is about $10 \%$, while the diversity within each genotype cluster is normally lower than $2 \%$ for genotype 1 and in the range 3-10\% for genotypes 2 and 3 [71]. All genotypes co-circulate, but with different frequencies and geographical distributions [72-74]. The prototype genotype 1 is ubiquitous worldwide and includes the greatest part of circulating virus, mainly referred to as subtype $1 \mathrm{a}$, while a subtype $1 \mathrm{~b}$ and other minor variants are rarer and confined to limited geographical areas. Genotype 2 appears to be the older variant with respect to genotypes 1, can 
be detected as the most common genotype in ancient tissues and is commonly harbored in tissues of elderly populations, while is only sporadically detected as circulating virus. Genotype 3, also an old variant divided in the two distinct subtypes $3 a$ and $3 b$, circulates at relatively higher frequencies in western Africa and at lower frequencies in other geographic areas. The three B19V genotypes share similar biological properties, pathogenic capacity, transmission routes, and pose a similar diagnostic challenge in the clinical setting. Hence, nucleic acid detection procedures should enable the detection and standardized quantification of all genotypes.

The pursuit of these main requirements can take advantage of an ample amount of sequence information from nucleotide databases, included that of international standards as reference materials for all genotypes [75]. However, the extent of sequence diversity that can be expected in the clinical isolates needs to be taken into account. For genotype 1 , available sequence information indicates a continuous evolutionary process, with a rather high mutation rate coupled to contrasting selection pressures on different genomic regions [76], but alignment algorithms still yield a consistent consensus sequence coupled with a limited genetic diversity within isolates collected over wide temporal and geographical ranges [77]. This low genetic diversity of genotype 1 suggests that primers and probes designed and used in molecular detection assays will recognize most targets with high probability. Genotypes 2 and 3 pose different problems. In both cases, fewer genomic sequences are presently available, inter- and intra-genotypic diversity is higher, the consistency of consensus sequences is lower than for genotype 1 , and it may be expected that addition of new sequences may demonstrate additional genetic diversity. This higher sequence divergence may pose diagnostic challenges, and primers and probes may recognize targets with lower probability.

Considerations on genotype distribution and sequence heterogeneity among isolates should guide the design of primers and probes for a molecular amplification assay [78-80]. Sequence alignment permits the definition of consensus sequences for each genotype, and of a whole-species consensus that can be used to define positions of amplification primers and probes. However, any choice of primers and probes does not exclude the possibility of mismatches to individual clinical isolates, causing impaired annealing and leading to underestimation or misdetection of targets. In well-designed assays, single base mismatches can be present either on one of the primer binding sites, or on the probe binding site, at an expected frequency lower than $1 \%$. Dual targeting assays are an alternative to minimize the risk of mismatching and underreporting positive samples [81].

Analytical performance. Different qPCR protocols have been developed to allow choice with respect to operational systems and diagnostic requirements. Consensus genotype-independent detection of B19V DNA by means of intercalating dyes may be an alternative to genotype specific detection by means of fluorescence probes, or the two detection formats may be successfully combined maintaining equal sensitivity and specificity of the assay. This latter scheme would guarantee a specific identification of prototype or variant genotypes coupled to a high flexibility in 
the detection of newly-emerging variants with possible additional sequence heterogeneity [78]. Analytical performance of the designed molecular assay, both in house as well as commercially available, should be evaluated according to guidelines for harmonization in analytical assays towards the international panel of standard reference material. Analytical requirements need to be met in terms of limit of detection and quantification, and linear range of the assay, and results reported in International Units (IU) rather than genome copies. Calibration of the assay in IU, as defined by WHO standards (1st WHO International Reference Panel for Parvovirus B19 Genotypes for NAT based assays NIBSC code: 09/110; and the related 3rd WHO International Standard for B19V, NIBSC 12/208), will reduce inter-lab heterogeneity in reporting of results and facilitate comparison of data collected in different experimental settings. Concerning assay setup, a useful and physically meaningful lower limit of detection or quantification should be set at $95 \%$ confidence in the range $10^{2}-10^{3} \mathrm{IU} / \mathrm{mL}$, while the range of linearity of the assay should extend to at least from $10^{3} \mathrm{IU} / \mathrm{mL}$ up to $10^{9} \mathrm{IU} / \mathrm{mL}$. Diagnostic laboratories can rely on commercially-available validated diagnostic assays. However, diagnostic kits encounter problems due to target heterogeneity, and for B19V some reports and proficiency panel evaluations suggest that these qPCR assays may not always conform to analytical performance standards [82]. Conversely, the in-house development of molecular assays may allow a wider operational choice but requires a high degree of conformity to analytical guidelines and validation procedures to ensure reliable results. It is worth noting that there are relatively few published reports on the analytical validation of molecular assays aimed at detecting B19V DNA, and mainly in the context of blood safety issues [81, 83]. Information on the analytical and clinical performance of molecular diagnostic assays would indeed prove extremely useful in the context of diagnostic laboratories.

\subsection{In Situ Hybridization Techniques}

A useful complement to qPCR is offered by in situ hybridization for the detection of viral nucleic acids within cells or tissues, with preservation of cellular morphology. In the case of biopsies, the quantitative information offered by qPCR on purified nucleic acids should not be considered of primary relevance, even if standardized by a reference endogenous target, because of the variability inherent in the sampling of a non-uniform cell population with different degrees of permissiveness to viral replication or different spread of virus. By in situ hybridization techniques, infected cells can be easily identified in the sampled material and give indication on the distribution of infection within tissues, and to what cellular types are involved. Several methodologies have been developed, differing in the choice of probe (DNA, oligo-DNA, or oligo-PNA probes), labeling method and moiety, and detection method. Immunofluorescence or immunoenzymatic detection methods can be successfully used. In the latter case, chromogenic substrates offer the advantage of an easy microscopic inspection of the analyzed tissue, while the use of chemiluminescent substrates may offer the advantage of a quantitative assessment of the abundance of targets within infected cells [84], although at the expense of sophisticated equipment. Coupling of 
fluorescent in situ hybridization techniques to cytofluorimetric cell population analysis is also feasible and can yield valuable information on the distribution of infected cells in a complex cell population [85], but it is technically demanding and its application in a diagnostic setting will require further standardization.

\subsection{Antigen Detection Techniques}

Immunological detection of viral proteins is not advisable for detection of virus in blood, unless for low-sensitivity screenings [86], but can be considered as an useful tool for identification of productively infected cells in tissue samples and a complement to in situ hybridization techniques. Commercially available monoclonal or polyclonal antibodies are directed against the viral capsid proteins, mostly towards the VP1/VP2 common epitopes. In selected applications, mainly when investigating the presence, expression and role of B19V in atypical contexts, in situ hybridization techniques, immunologic detection of viral proteins, and/or of cellular markers, might be combined to better characterize a productive viral infection and identify the phenotype of target cells [11].

\subsection{Antibody detection assays}

Detection of a specific immune response by the presence of anti-B19V antibodies specific to B19V capsid antigens is still now considered the standard and most widely used means of laboratory diagnosis of B19V infection. Indirect markers of active viral infection have recently been identified, such as the production of chemokine CXCL10 [87], or high neopterin levels indicative of a cellmediated immune response [30], however parallel detection of specific anti-B19 IgM and IgG antibodies is required and interpretation of the combination of results may allow for a presumptive diagnosis of active, recent or past infection [88]. VP antigens used for immunological detection are obtained by means of heterologous recombinant expression systems and, depending on their characteristics and assay setup, can offer different information useful for interpretation of a specific immune response $[79,89]$. Recombinant proteins expressed in prokaryotic systems lose their native conformation and are suitable for the detection of immunity against linear epitopes, while recombinant proteins expressed in eukaryotic system can maintain native conformation and can be used to detect immunity against conformational epitopes [90-92]. In particular, viral capsid proteins assemble as VLPs with antigenic configuration quite similar to that on native virus and are the recognized standard for immunological detection. Commercially available enzyme immunoassays, or chemiluminescent immunoassays, can use VLPs composed of VP2 only, or VP2+VP1, or VP2+VP1 expressed in prokaryotic systems, to allow detection of antibodies to conformational VP2 or also VP1 linear epitopes. Available Line blot assays include an array of conformational and linear antigens, and can be used as a confirmatory assay to dissect the range of antibody response to $\mathrm{B} 19 \mathrm{~V}$. Of limited availability and not widely used, although potentially useful to determine the timing with respect to onset of infection, are assays to determine IgG avidity [93], or acute-phase, epitope specific reactivity [94]. Recent technical developments aimed at the setup of 
homogenous phase, multiplexed assays, first proposed the use of array-in-well $\lg G$ antibody detection, coupled to photon emitting nanoparticles and photoluminescence reporter technology [95], then a Luminex-based assay for the detection of specific lgG, potentially combined in a multiplex assay aimed at antenatal screening [96].

Given the scarcity of comparative evaluation of tests as reported in the recent literature [79, 97], the operative choice within currently available test formats and platforms is open to different and somewhat subjective evaluations, and comparison of results obtained with different assays is uncertain. The analytical interpretation of test results is suggested by the manufacturers, but also considering the possibility of falsely reactive results frequently due to heterologous polyclonal activation or antigenic cross reactivity, the clinical interpretation of analytical results requires a more cautious and independent approach to identify a meaningful clinical cut-off.

\subsection{Future developments}

Apart from qPCR, other molecular amplification techniques and detection formats have not maintained a reliable utility in the diagnostic laboratories, and have not been reported in the scientific literature in the context of an extended clinical use. However, some technical improvements can still be pursued, in a double direction. First, towards the development of dedicated point-of care, lab-on-chip devices, able to couple amplification and detection of molecular targets on stand-alone equipment $[98,99]$. Then, towards the inclusion of B19V as target in innovative array-based assays, that can couple amplification and detection in a format enabling identification of its presence within an ample set of possible infectious agents [100-104]. Implementation of such assays in the current diagnostic algorithms remains at present a stimulating challenge.

Next generation sequencing methodologies are increasingly applied to investigation of clinical samples, and metagenomic investigations can be aimed at the identification of viral sequences in either blood or solid tissues. B19V genomic sequences have been detected in blood as part of the plasma virome in normal population [31], in peculiar epidemiological and clinical contexts [105], or in situations with underlying pathological process in the quest for etiological infectious agents [106108]. This kind of experimental evidence is only now becoming to accumulate and will likely provide valuable information on the presence and relevance of $\mathrm{B} 19 \mathrm{~V}$ in many pathological processes. Presently, independently of the technique employed, it is useful to remind that the mere presence of viral genomic sequences, or even viral messengers, is by itself insufficient to claim for any causal role of $\mathrm{B} 19 \mathrm{~V}$ in a pathogenetic process, unless linked by a definite relationship to observed alterations.

\section{Conclusion}


$\mathrm{B} 19 \mathrm{~V}$ is a possible etiological agent in a large ensemble of diseases, with a main target on erythroid progenitor cells in bone marrow but encompassing practically all tissues and organs, intrauterine infections included. An extended awareness and definition of the actual pathogenic role of $\mathrm{B} 19 \mathrm{~V}$ among human diseases will be fostered by the development of better diagnostic methods and algorithms. In this respect, integration of the common immunological diagnostic scheme with the application of molecular detection methods will be crucial. Molecular detection of $\mathrm{B} 19 \mathrm{~V}$ is easy and reliable. qPCR techniques for the detection of B19V can be carried out from biological specimens with standard pre-analytical processing procedures. The choice of qPCR techniques range from commercially-available, to integrated in common-flow analytical platforms, to the tailored or lab-developed assays, requiring standard laboratory equipment. Development of even more versatile analytical platforms, of alternative molecular assays with point-of-care characteristics, will widen in the future the opportunities for molecular testing for B19V, aimed at improving performance and informative content, and reducing time and costs. The pattern of B19V genetic evolution and diversity, its biological characteristics and complex relationship with the host, its diverse clinical manifestations of infection, are all topics that are far from completely understood, and will benefit from information emerging from the wider use of molecular diagnostic approaches and development of innovative methodologies. 


\section{Expert commentary}

Given the complex scenario of the infectious process and the diversity in possible pathogenetic mechanisms, an accurate and informative laboratory diagnosis of B19V infection necessarily relies on a multi-parametric approach, combining as much as possible the molecular detection of viral components, mainly viral DNA, to the immunological detection of virus-specific antibodies. A laboratory diagnosis is necessary to confirm or exclude B19V infection, to differentiate acute from persistent infections, and in the follow-up of documented infections. Investigation to document a B19V infection can be rather straightforward, or be part of complex diagnostic workouts in atypical clinical settings. Moreover, B19V detection can be part of screening procedures, especially considering antenatal screening, or part of prophylactic measures to prevent iatrogenic transmission of virus. All of these diagnostic needs will take advantage of an integrated molecular and immunological approach and require a critical assessment of laboratory findings. In Figure 2, the analytical profile obtained in a set of serum samples by parallel detection of anti-B19V IgM and IgG antibodies, and of viral DNA by qPCR, is reported, highlighting the complex picture that can be obtained when investigating for a B19V infection and the consequent necessity of integration of molecular and immunological data for a correct and timely diagnosis.

Practically, the indirect, immunological approach to the diagnosis of B19V infection is commonly followed as a first level investigation. The acute phase of infection is characterized by high viremic load and the emergence of specific $\operatorname{lgM} / \mathrm{lgG}$ antibodies and is normally followed by a progressive clearance of viremia still in the presence of specific IgM and IgG, or may lead to persistent infections, usually in the presence of IgG only, and finally may result in the silent persistence of virus in tissues. Thus, coupled determination of $\lg \mathrm{G} / \mathrm{lgM}$ antibodies against VP proteins is required. The presence of IgM reactivity is indicative of an active or recent infection, and a repeated determination can be used as a confirmatory assay. In addition, assays able to discriminate a differential reactivity of $\operatorname{lgG}$ against conformational or linear epitopes can be used to confirm or better characterize the immunological response. However, the immunological approach presents intrinsic limitations that prompt molecular diagnostic testing.

Indications for molecular testing include the confirmation of suspected active infections, the followup of the course of documented infections, and the characterization of persistent infections. Molecular analysis is normally carried out from peripheral blood since productive infections are characterized by viremia, and viremic levels are correlated with the clinical course. Both serum and plasma are suitable samples, so that detection of both viral genomes and specific antibodies can be obtained from the same sample. The highest viremic levels are reached in the initial phase of infection, before or coupled to the development of a detectable immune response. In this case, a single immunological determination may yield unreliable results, while repeated testing will lead to a delay in diagnosis. In a later phase of infection, progressively lowering viral loads can be 
detected in the presence of both IgG and IgM. Clearance of viremia might be delayed and parallel the waning of IgM, but in persistent infections even sustained levels of viremia can be present when IgM are no longer detected. In immunodeficient, immunosuppressed, or in transfused (and IVIG-treated) patients, antibody detection is just unreliable for diagnostic purposes and detection of viral DNA should be considered the only relevant diagnostic parameter. In antenatal screening or prenatal diagnosis, relying on maternal immune status only will lead to underestimation of active infections in the mother and correlated risk to fetus, so detection of viral DNA in maternal blood should be part of the diagnostic workup.

In addition to determination of the immune status of infected individuals, molecular amplification methods leading to a reliable quantitative assessment of viral load in an ample dynamic range are critically required to obtain useful diagnostic information. In fact, considering peripheral blood as the sample of choice and the characteristics of a typical time course of infection, a high viral load in the acute phase will progressively decline to lower levels in the following months, but even in normal subjects a complete clearance of B19V DNA from peripheral blood may not be achieved in a short time. Therefore, the diagnostic question will be to discriminate this normal situation from an active, persistent infection and determination of the viral load in the course of time is the only relevant parameter for this purpose, with the recently realized caveat that target viral DNA may be in the form of free DNA and not directly correlated to a persistent productive infection. In pregnant women, either in case of antenatal screening or in response to a specific diagnostic question, determination of B19V DNA is a more reliable marker of infection than determination of the immunological status, while confirmation of intrauterine or congenital infection necessarily relies on the detection of B19V DNA in fetal or neonatal samples. It is important that all viral genotypes are detected by a molecular assay with the same analytical sensitivity, although genotyping can be a secondary issue with respect to diagnosis. Sequencing of amplification products might be carried mainly for epidemiological studies, especially to confirm the presence and identity of variant genotypes, but its clinical utility still remains questionable until some sound correlation emerges between a particular sequence variant and peculiar pathological processes, which has not been the case until now.

In case of predominant hematological involvement the virus can also be detected from bone marrow aspirates or biopsies, where molecular detection methods should be performed in addition to histologic examination. Biopsy samples can be obtained from other tissues where an active B19V infection is suspected, for example in the case of acute myocarditis, and in these instances quantitative molecular assays will be useful in the differentiation between an active infection or the mere persistence of viral genomes. Molecular analysis are necessary to confirm infection of the fetus, and for this purpose it is not necessary to analyze cord blood, since the virus can also be detected in amniotic fluid, or in placenta at term, or in biopsies in the case of post-mortem analysis. In all these situations, whenever possible, GPCR assays should be usefully complemented by in 
situ hybridization or antigen detection techniques to yield information not only on the presence of virus, but also on its expression and the extent of infection in the different cell types within the sampled tissue.

\section{Five-year view}

B19V is still underestimated from a clinical perspective. Its wide circulation and prevalent benign and self-limiting clinical course generally lead to a diminished appreciation of its pathogenic potential. However, B19V is a relevant human pathogenic virus, and an effort should be undertaken to improve diagnostic awareness and appropriateness. Integration of the common immunological diagnostic scheme with the application of molecular detection methods should be pursued whenever possible, given that both antibody detection and molecular assays are available as commercial tests, in some cases already integrated on analytical platforms. Benchmark analysis should be presented to assist in operational choice with respect to different available tests. In the future, important information on the role of $\mathrm{B} 19 \mathrm{~V}$ in the development of pathological process is expected from application of NGS techniques directed at analyzing the metagenomic structure and expression patterns in diseased tissues, and this may translate in the individuation of relevant diagnostic markers, useful in a clinical setting not only for diagnosis but also for therapeutic management.

\section{Funding}

This paper is not funded.

\section{Declaration of interest}

The author has no relevant affiliations or financial involvement with any organization or entity with a financial interest in or financial conflict with the subject matter or materials discussed in the manuscript. This includes employment, consultancies, honoraria, stock ownership or options, expert testimony, grants or patents received or pending, or royalties. 


\section{Key issues}

- Parvovirus B19 (B19V), a ssDNA in the family Parvoviridae, is a widely circulating human pathogenic virus, implicated in a complex relationship with the host and ample range of clinical manifestations, posing the necessity of diagnostic awareness and appropriateness.

- $\quad$ B19V shows a selective tropism for erythroid progenitor cells, productive infection causes a temporary block in erythropoiesis that can manifest as a transient or persistent erythroid aplasia. The role of the immune system in controlling infection is crucial.

- In non-erythroid tissues, including endothelial, stromal, or synovial cells, infection is usually abortive and the virus is supposed to exert its pathological potential by indirect mechanisms, such as the induction of inflammatory or autoimmune processes.

- $\quad$ The virus can persist in diverse tissues, so the mere detection viral DNA cannot be assumed as a diagnostic criteria sufficient for assessment of a direct pathogenetic role.

- $\quad$ The virus can be transmitted from mother to fetus, posing a risk of fetal damage that should prompt for an antenatal assessment of risk of fetal infections and diagnostic attention towards the development of intrauterine infections.

- The range of molecular targets relevant for a direct diagnosis of infection is restricted mainly to viral DNA. qPCR is the method of choice, and in the diversity of proposed assays two main requirements should be met: (i) the capability of detection of all genotypes of B19V, and (ii) a calibrated and standardized quantification of target.

- $\quad$ GPCR can be useful complemented by in situ hybridization detection methods for the detection of viral nucleic acids within cells or tissues, and/or by immunologic detection of viral proteins, to better characterize a productive viral infection and identify the phenotype of target cells.

- $\quad$ Detection of a specific immune response by the presence of anti-B19V antibodies is considered the standard and most widely used means of laboratory diagnosis of B19V infection. Parallel detection of specific anti-B19 IgM and IgG antibodies is required and interpretation of the combination of results may allow for a presumptive diagnosis of active, recent or past infection.

- $\quad$ Given the complex scenario of the infectious process and the diversity in possible pathogenetic mechanisms, an accurate and informative laboratory diagnosis of B19V infection necessarily relies on a multi-parametric approach, combining as much as possible the molecular detection of viral components, mainly viral DNA, to the detection of virusspecific antibodies. 


\section{Figure Legends}

Figure 1. B19V genome organization and virion structure.

Top: coding sequences for the viral proteins: NS, non-structural protein; VP, structural proteins, colinear VP1 and VP2, assembled in a T=1 icosahedral capsid; $7.5 \mathrm{kDa}, 9.0 \mathrm{kDa}, 11 \mathrm{kDa}$ : minor non-structural proteins.

Center: a schematic diagram of B19V genome indicating the two inverted terminal regions (ITR), and the internal region (IR) with the distribution of cis-acting functional sites (P6, promoter; pAp1, pAp2, proximal cleavage-polyadenylation sites; pAd, distal cleavage-polyadenylation site; D1, D2, splice donor sites; A1.1, A1.2, A2.1, A2.2, splice acceptor sites).

Bottom: simplified transcription map of B19V genome. Five classes of mRNAs (mRNA 1-5) include a total of 12 different mRNAs species, arising from alternative usage of splicing and cleavage signals, as indicated by dashed lines, with different coding capacity for the main B19V proteins.

Figure 2.

Analytical profile of a group of serum samples sent to the diagnostic virology laboratory of S.Orsola-Malpighi Hospital, University of Bologna, for a diagnosis of B19V infection. Samples were concurrently tested for the detection of B19V specific $\lg G$ and IgM antibodies by a VLP-based CLIA assay (Liaison XL, DiaSorin, Italy), and by qPCR for the detection of B19V DNA and determination of viral load [78]. Out of 293 samples, $53(18 \%)$ resulted positive for the presence of B19V DNA, confirming active infection. Of these, 36 had IgM values $>1.0$, and $29 \operatorname{lgM}$ values $>3.0$. Viral load ranged from $10^{1} \mathrm{IU} / \mathrm{mL}$ up to $10^{10} \mathrm{IU} / \mathrm{mL}$ (IU: NIBSC international units). The ample dynamic range of CLIA assay requires a careful consideration of cut-off values, especially when considering low-level IgM positive results. QPCR confirms its essential role to discriminate active infections in case of low-level lgM results and in the detection of chronic infections. 


\section{References}

Papers of special note have been highlighted as either of interest $(\cdot)$ or of considerable interest $(\bullet)$ to readers.

1. Cotmore SF, Agbandje-McKenna M, Chiorini JA, Mukha DV, Pintel DJ, Qiu J, SoderlundVenermo M, Tattersall P, Tijssen P, Gatherer D, Davison AJ. The family Parvoviridae. Arch Virol (2014) 159:1239-47.

2. Gallinella G. Parvovirus B19 Achievements and Challenges. ISRN Virology (2013):10.5402/2013/898730.

${ }^{*}$ A comprehensive critical review on Parvovirus B19

3. Qiu J, Soderlund-Venermo M, Young NS. Human Parvoviruses. Clin Microbiol Rev (2017) 30:43-113.

${ }^{*}$ A comprehensive critical review on all viruses in the family Parvoviridae, able to infect humans

4. Leisi R, Ruprecht N, Kempf C, Ros C. Parvovirus B19 uptake is a highly selective process controlled by VP1u, a novel determinant of viral tropism. J Virol (2013) 87:13161-7.

5. Leisi R, Von Nordheim M, Ros C, Kempf C. The VP1u Receptor Restricts Parvovirus B19 Uptake to Permissive Erythroid Cells. Viruses (2016) 8.

6. Luo Y, Qiu J. Human parvovirus B19: a mechanistic overview of infection and DNA replication. Future Virol (2015) 10:155-167.

7. Bua G, Manaresi E, Bonvicini F, Gallinella G. Parvovirus B19 Replication and Expression in Differentiating Erythroid Progenitor Cells. PLoS One (2016) 11:e0148547.

* A detailed description of replication and expression of B19V in primary target cells, and a framework for intepreting molecular data in view of a productive or abortive viral lifecycle

8. Ganaie SS, Quu J. Recent Advances in Replication and Infection of Human Parvovirus B19. Front Cell Infect Microbiol (2018) 8:166.

* A recent, concise but comprehensive review on virus-cell interactions at the molecular level

9. Ganaie SS, Zou W, Xu P, Deng X, Kleiboeker S, Qiu J. Phosphorylated STAT5 directly facilitates parvovirus B19 DNA replication in human erythroid progenitors through interaction with the MCM complex. PLoS Pathog (2017) 13:e1006370.

* How Parvovirus B19 is able to convert cellular machinery to a viral DNA replicative complex

10. Xu P, Zhou Z, Xiong M, Zou W, Deng X, Ganaie SS, Kleiboeker S, Peng J, Liu K, Wang S, Ye SQ, Qiu J. Parvovirus B19 NS1 protein induces cell cycle arrest at G2-phase by activating the ATR-CDC25C-CDK1 pathway. PLoS Pathog (2017) 13:e1006266.

* How Parvovirus B19 is capable of interacting with the cellular machinery and subvert regulation of the cell cycle

11. Adamson-Small LA, Ignatovich IV, Laemmerhirt MG, Hobbs JA. Persistent parvovirus B19 infection in non-erythroid tissues: possible role in the inflammatory and disease process. Virus Res (2014) 190:8-16.

* A comprehensive critical review on available information on the pathogenetic role and persistence of parvovirus B19 in non-erytrhoid tissues, with interesting considerations on the diagnostic criteria

12. Bua G, Gallinella G. How does parvovirus B19 DNA achieve lifelong persistence in human cells? Future Virology (2017) 12:549-553. 
13. De Cao E, Zagheni E, Manfredi P, Melegaro A. The relative importance of frequency of contacts and duration of exposure for the spread of directly transmitted infections. Biostatistics (2014) 15:470-83.

14. Abrams S, Hens N. Modeling individual heterogeneity in the acquisition of recurrent infections: an application to parvovirus B19. Biostatistics (2015) 16:129-42.

15. Goeyvaerts N, Hens N, Aerts M, Beutels P. Model structure analysis to estimate basic immunological processes and maternal risk for parvovirus B19. Biostatistics (2011) 12:283302.

16. Bonvicini F, Bua G, Gallinella G. Parvovirus B19 infection in pregnancy-awareness and opportunities. Curr Opin Virol (2017) 27:8-14.

17. Bascietto F, Liberati M, Murgano D, Buca D, lacovelli A, Flacco ME, Manzoli L, Familiari A, Scambia G, D'Antonio F. Outcomes associated with fetal Parvovirus B19 infection: A systematic review and meta-analysis. Ultrasound Obstet Gynecol (2018).

18. Ornoy A, Ergaz Z. Parvovirus B19 infection during pregnancy and risks to the fetus. Birth Defects Res (2017) 109:311-323.

19. Marano G, Vaglio S, Pupella S, Facco G, Calizzani G, Candura F, Liumbruno GM, Grazzini G. Human Parvovirus B19 and blood product safety: a tale of twenty years of improvements. Blood Transfus (2015) 13:184-96.

20. Juhl D, Hennig H. Parvovirus B19: What Is the Relevance in Transfusion Medicine? Front Med (Lausanne) (2018) 5:4.

21. Kerr JR. A review of blood diseases and cytopenias associated with human parvovirus B19 infection. Rev Med Virol (2015) 25:224-40.

* A comprehensive critical review on the impact of parvovirus B19 on the diverse blood cell lineages, and associated clinical manifestations

22. Ganzel C, Constantin R. Parvovirus B19 diagnosed by bone marrow biopsy. Blood (2015) 125:3351.

23. Rogers HJ, Feasel P. Acute parvovirus B19 infection detected in bone marrow biopsy. Blood (2015) 126:1630.

24. Means RT, Jr. Pure red cell aplasia. Blood (2016) 128:2504-2509.

25. Tsitsikas DA, Gallinella G, Patel S, Seligman H, Greaves P, Amos RJ. Bone marrow necrosis and fat embolism syndrome in sickle cell disease: increased susceptibility of patients with non-SS genotypes and a possible association with human parvovirus B19 infection. Blood Rev (2014) 28:23-30.

26. Verdonschot J, Hazebroek M, Merken J, Debing Y, Dennert R, Brunner-La Rocca HP, Heymans S. Relevance of cardiac parvovirus B19 in myocarditis and dilated cardiomyopathy: review of the literature. Eur J Heart Fail (2016) 18:1430-1441.

* A comprehensive critical review on the relevance of Parvovirus B19 in myocarditis and myocardiopathies, a highly controversial issue. Caveat: in the review (and in some of the literature on this topic) it is sometimes referred to viral mRNA as 'replicative intermediate', which is not correct since B19V is not a reverse-transcribing virus. Implication of detecting viral RNA change if this non-correct assumption is maintained.

27. Kerr JR. The role of parvovirus B19 in the pathogenesis of autoimmunity and autoimmune disease. J Clin Pathol (2016) 69:279-91.

* A comprehensive critical review on the role and possible mechanisms involving parvovirus B19 in autoimmune diseases

28. Thammasri K, Rauhamaki S, Wang L, Filippou A, Kivovich V, Marjomaki V, Naides SJ, Gilbert L. Human parvovirus B19 induced apoptotic bodies contain altered self-antigens that are phagocytosed by antigen presenting cells. PLoS One (2013) 8:e67179. 
29. Juhl D, Gorg S, Hennig H. Persistence of Parvovirus B19 (B19V) DNA and humoral immune response in B19V-infected blood donors. Vox Sang (2014) 107:226-32.

30. Bonjoch X, Obispo F, Alemany C, Pacha A, Rodriguez E, Xairo D. Characterization of Markers of the Progression of Human Parvovirus B19 Infection in Virus DNA-Positive Plasma Samples. Transfus Med Hemother (2015) 42:233-8.

31. Moustafa A, Xie C, Kirkness E, Biggs W, Wong E, Turpaz Y, Bloom K, Delwart E, Nelson KE, Venter JC, Telenti A. The blood DNA virome in 8,000 humans. PLoS Pathog (2017) 13:e1006292.

* NGS techniques for the detection of DNA viruses in blood, first steps toward a future virlogical paradigm

32. Jain A, Jain P, Prakash S, Kumar A, Khan DN, Seth A, Gupta S, Kant R. Genotype 3b of human parvovirus B19 detected from hospitalized children with solid malignancies in a North Indian tertiary care hospital. J Med Virol (2016) 88:1922-9.

33. Jain A, Jain P, Kumar A, Prakash S, Khan DN, Kant R. Incidence and progression of Parvovirus B19 infection and molecular changes in circulating B19V strains in children with haematological malignancy: A follow up study. Infect Genet Evol (2018) 57:177-184.

34. Rahiala J, Koskenvuo M, Norja P, Meriluoto M, Toppinen M, Lahtinen A, Vaisanen E, Waris M, Vuorinen T, Saarinen-Pihkala U, Lappalainen M, Allander T, Ruuskanen O, Hedman K, Soderlund-Venermo M, Vettenranta K. Human parvoviruses B19, PARV4 and bocavirus in pediatric patients with allogeneic hematopoietic SCT. Bone Marrow Transplant (2013) 48:1308-12.

35. Ohrmalm L, Gustafson I, Lindblom A, Norbeck O, Johansson JE, Brune M, Ljungman P, Broliden K. Human parvovirus B19 in pediatric and adult recipients of allogeneic hematopoietic stem cell transplantation. Bone Marrow Transplant (2013) 48:1366-7.

36. Eid AJ, Chen SF, Practice ASTIDCo. Human parvovirus B19 in solid organ transplantation. Am J Transplant (2013) 13 Suppl 4:201-5.

37. Plentz A, Wurdinger M, Kudlich M, Modrow S. Low-level DNAemia of parvovirus B19 (genotypes 1-3) in adult transplant recipients is not associated with anaemia. J Clin Virol (2013) 58:443-8.

38. Porignaux R, Vuiblet V, Barbe C, Nguyen Y, Lavaud S, Toupance O, Andreoletti L, Rieu P, Leveque N. Frequent occurrence of parvovirus B19 DNAemia in the first year after kidney transplantation. J Med Virol (2013) 85:1115-21.

39. Wurdinger M, Modrow S, Plentz A. Impact of Parvovirus B19 Viremia in Liver Transplanted Children on Anemia: A Retrospective Study. Viruses (2017) 9.

40. Molenaar-de Backer MW, Russcher A, Kroes AC, Koppelman MH, Lanfermeijer M, Zaaijer HL. Detection of parvovirus B19 DNA in blood: Viruses or DNA remnants? J Clin Virol (2016) 84:19-23.

* How the introduction of a simple technical step may change analytical results and our perspective on virus-host relationship

41. Reber $U$, Moser $O$, Dilloo $D$, Eis-Hubinger AM. On the utility of the benzonase treatment for correct laboratory diagnosis of parvovirus B19 infection. J Clin Virol (2017) 95:10-11.

42. Pankuweit S, Stein A, Karatolios K, Richter A, Ruppert V, Maisch B. Viral genomes in the pericardial fluid and in peri- and epicardial biopsies from a German cohort of patients with large to moderate pericardial effusions. Heart Fail Rev (2013) 18:329-36.

43. Pankuweit $\mathrm{S}$, Klingel K. Viral myocarditis: from experimental models to molecular diagnosis in patients. Heart Fail Rev (2013) 18:683-702.

* A very accurate account on the role of viruses, including Parvovirus B19, in the development of myocarditis and myocardiopathies 
44. Maisch B, Pankuweit S. Standard and etiology-directed evidence-based therapies in myocarditis: state of the art and future perspectives. Heart Fail Rev (2013) 18:761-95.

45. Soderlund-Venermo M. Clinical significance of parvovirus B19 DNA in cutaneous biopsies. Br J Dermatol (2017) 177:900-901.

46. Santonja C, Santos-Briz A, Palmedo G, Kutzner H, Requena L. Detection of human parvovirus B19 DNA in $22 \%$ of 1815 cutaneous biopsies of a wide variety of dermatological conditions suggests viral persistence after primary infection and casts doubts on its pathogenic significance. Br J Dermatol (2017) 177:1060-1065.

47. Lecuit M, Eloit M. The human virome: new tools and concepts. Trends Microbiol (2013) 21:510-5.

48. Virgin HW. The virome in mammalian physiology and disease. Cell (2014) 157:142-50.

49. Rascovan N, Duraisamy R, Desnues C. Metagenomics and the Human Virome in Asymptomatic Individuals. Annu Rev Microbiol (2016) 70:125-41.

50. Toppinen M, Perdomo MF, Palo JU, Simmonds P, Lycett SJ, Soderlund-Venermo M, Sajantila A, Hedman K. Bones hold the key to DNA virus history and epidemiology. Sci Rep (2015) 5:17226.

* Evolution and persistence of parvovirus B19 in ancient tissues

51. Pyoria L, Toppinen M, Mantyla E, Hedman L, Aaltonen LM, Vihinen-Ranta M, Ilmarinen T, Soderlund-Venermo M, Hedman K, Perdomo MF. Extinct type of human parvovirus B19 persists in tonsillar B cells. Nat Commun (2017) 8:14930.

* Evolution and persistence of parvovirus B19 in unexpected cells and anatomical sites

52. Janovitz T, Wong S, Young NS, Oliveira T, Falck-Pedersen E. Parvovirus B19 integration into human CD36+ erythroid progenitor cells. Virology (2017) 511:40-48.

* First report of parvovirus B19 genome as an integrative element, although in vitro and in a labile cell population

53. Molina KM, Garcia X, Denfield SW, Fan Y, Morrow WR, Towbin JA, Frazier EA, Nelson DP. Parvovirus B19 myocarditis causes significant morbidity and mortality in children. Pediatr Cardiol (2013) 34:390-7.

54. Bock CT, Duchting A, Utta F, Brunner E, Sy BT, Klingel K, Lang F, Gawaz M, Felix SB, Kandolf R. Molecular phenotypes of human parvovirus B19 in patients with myocarditis. World J Cardiol (2014) 6:183-95.

55. Niccoli G, Severino A, Pieroni M, Cosentino N, Ventrone MA, Conte M, Roberto M, Gallinella G, Liuzzo G, Leone AM, Porto I, Burzotta F, Trani C, Crea F. Parvovirus B19 at the culprit coronary stenosis predicts outcome after stenting. Eur J Clin Invest (2014) 44:209-18.

56. Greulich S, Kindermann I, Schumm J, Perne A, Birkmeier S, Grun S, Ong P, Schaufele T, Klingel K, Schneider S, Kandolf R, Bohm M, Sechtem U, Mahrholdt H. Predictors of outcome in patients with parvovirus B19 positive endomyocardial biopsy. Clin Res Cardiol (2016) 105:37-52.

57. Kuhl U, Lassner D, Dorner A, Rohde M, Escher F, Seeberg B, Hertel E, Tschope C, Skurk C, Gross UM, Schultheiss HP, Poller W. A distinct subgroup of cardiomyopathy patients characterized by transcriptionally active cardiotropic erythrovirus and altered cardiac gene expression. Basic Res Cardiol (2013) 108:372.

58. Bachelier K, Biehl S, Schwarz V, Kindermann I, Kandolf R, Sauter M, Ukena C, Yilmaz A, Sliwa K, Bock CT, Klingel K, Bohm M. Parvovirus B19-induced vascular damage in the heart is associated with elevated circulating endothelial microparticles. PLoS One (2017) 12:e0176311. 
59. Puccetti C, Contoli M, Bonvicini F, Cervi F, Simonazzi G, Gallinella G, Murano P, Farina A, Guerra B, Zerbini M, Rizzo N. Parvovirus B19 in pregnancy: possible consequences of vertical transmission. Prenat Diagn (2012) 32:897-902.

60. Lindenburg IT, van Kamp IL, Oepkes D. Intrauterine blood transfusion: current indications and associated risks. Fetal Diagn Ther (2014) 36:263-71.

61. Lassen J, Bager P, Wohlfahrt J, Bottiger B, Melbye M. Parvovirus B19 infection in pregnancy and subsequent morbidity and mortality in offspring. Int J Epidemiol (2013) 42:1070-6.

62. Lindenburg IT, van Klink JM, Smits-Wintjens VE, van Kamp IL, Oepkes D, Lopriore E. Long-term neurodevelopmental and cardiovascular outcome after intrauterine transfusions for fetal anaemia: a review. Prenat Diagn (2013) 33:815-22.

63. Crane J, Mundle W, Boucoiran I, Maternal Fetal Medicine C. Parvovirus B19 infection in pregnancy. J Obstet Gynaecol Can (2014) 36:1107-1116.

64. Poliquin V, Yudin MH, Murphy KE, Okun N. Antepartum Screening for Maternal Infection and Immune Status: Is it Time to Broaden Our Routine? J Obstet Gynaecol Can (2015) 37:1118-21.

65. Watt AP, Brown M, Pathiraja M, Anbazhagan A, Coyle PV. The lack of routine surveillance of Parvovirus B19 infection in pregnancy prevents an accurate understanding of this regular cause of fetal loss and the risks posed by occupational exposure. J Med Microbiol (2013) 62:86-92.

66. Barlinn R, Rollag H, Trogstad L, Vainio K, Basset C, Magnus P, Dudman SG. High incidence of maternal parvovirus B19 infection in a large unselected population-based pregnancy cohort in Norway. J Clin Virol (2017) 94:57-62.

67. Bonvicini F, Manaresi E, Gallinella G, Gentilomi GA, Musiani M, Zerbini M. Diagnosis of fetal parvovirus B19 infection: value of virological assays in fetal specimens. BJOG (2009) 116:813-7.

68. Bonvicini F, Puccetti C, Salfi NC, Guerra B, Gallinella G, Rizzo N, Zerbini M. Gestational and fetal outcomes in B19 maternal infection: a problem of diagnosis. J Clin Microbiol (2011) 49:3514-8.

69. Hellmund A, Geipel A, Berg C, Bald R, Gembruch U. Early Intrauterine Transfusion in Fetuses with Severe Anemia Caused by Parvovirus B19 Infection. Fetal Diagn Ther (2018) 43:129-137.

70. Baylis SA, Ma L, Padley DJ, Heath AB, Yu MW, Collaborative Study G. Collaborative study to establish a World Health Organization International genotype panel for parvovirus B19 DNA nucleic acid amplification technology (NAT)-based assays. Vox Sang (2012) 102:20411.

71. Gallinella G, Venturoli S, Manaresi E, Musiani M, Zerbini M. B19 virus genome diversity: epidemiological and clinical correlations. J Clin Virol (2003) 28:1-13.

72. Hubschen JM, Minneva Z, Mentis AF, Schneider F, Aboudy Y, Grossman Z, Rudich H, Kasymbekova K, Sarv I, Nedeljkovic J, Tahita MC, Tarnagda Z, Ouedraogo JB, Gerasimova AG, Moskaleva TN, Tikhonova NT, Chitadze N, Forbi JC, Faneye AO, Otegbayo JA, Charpentier E, Muller CP. Phylogenetic analysis of human parvovirus B19 sequences from eleven different countries confirms the predominance of genotype 1 and suggests the spread of genotype 3b. J Clin Microbiol (2009) 47:3735-8.

73. Corcoran C, Hardie D, Yeats J, Smuts H. Genetic variants of human parvovirus B19 in South Africa: cocirculation of three genotypes and identification of a novel subtype of genotype 1. J Clin Microbiol (2010) 48:137-42.

74. Eis-Hubinger AM, Reber U, Edelmann A, Kalus U, Hofmann J. Parvovirus B19 genotype 2 in blood donations. Transfusion (2014) 54:1682-4. 
75. Trosemeier JH, Branting A, Lukashov VV, Blumel J, Baylis SA. Genome sequences of parvovirus b19 reference strains. Genome Announc (2014) 2.

76. Stamenkovic GG, Cirkovic VS, Siljic MM, Blagojevic JV, Knezevic AM, Joksic ID, Stanojevic MP. Substitution rate and natural selection in parvovirus B19. Sci Rep (2016) 6:35759.

77. Manaresi E, Conti I, Bua G, Bonvicini F, Gallinella G. A Parvovirus B19 synthetic genome: sequence features and functional competence. Virology (2017) 508:54-62.

78. Bonvicini F, Manaresi E, Bua G, Venturoli S, Gallinella G. Keeping pace with parvovirus B19 genetic variability: a multiplex genotype-specific quantitative PCR assay. J Clin Microbiol (2013) 51:3753-9.

* In house developed qPCR assay, based on a selected sequence dataset of parovirus B19 genotypes, with detection format flexibility and validated against international standards

79. Maple PA, Hedman L, Dhanilall P, Kantola K, Nurmi V, Soderlund-Venermo M, Brown KE, Hedman K. Identification of past and recent parvovirus B19 infection in immunocompetent individuals by quantitative PCR and enzyme immunoassays: a dual-laboratory study. J Clin Microbiol (2014) 52:947-56.

* How the integration of different information obtained from molecular and immunological assays are necessary to obtain an accurate diagnosis of parvovirus B19 infection

80. Toppinen M, Norja P, Aaltonen LM, Wessberg S, Hedman L, Soderlund-Venermo M, Hedman K. A new quantitative PCR for human parvovirus B19 genotypes. J Virol Methods (2015) 218:40-5.

* In house developed qPCR assay, based on a selected sequence dataset of parovirus B19 genotypes, with detection format flexibility and validated against international standards

81. Molenaar-de Backer MW, de Waal M, Sjerps MC, Koppelman MH. Validation of new realtime polymerase chain reaction assays for detection of hepatitis $A$ virus RNA and parvovirus B19 DNA. Transfusion (2016) 56:440-8.

82. Pisani G, Cristiano K, Fabi S, Simeoni M, Marino F, Gaggioli A. A significantly lower potency observed for the 3rd WHO International Standard for Parvovirus B19V DNA with the cobas TaqScreen DPX test. Vox Sang (2016) 111:115-9.

83. Muller MM, Fraile MI, Hourfar MK, Peris LB, Sireis W, Rubin MG, Lopez EM, Rodriguez GT, Seifried E, Saldanha J, Schmidt M. Evaluation of two, commercial, multi-dye, nucleic acid amplification technology tests, for HBV/HCV/HIV-1/HIV-2 and B19V/HAV, for screening blood and plasma for further manufacture. Vox Sang (2013) 104:19-29.

84. Bonvicini F, Mirasoli M, Manaresi E, Bua G, Calabria D, Roda A, Gallinella G. Single-cell chemiluminescence imaging of parvovirus B19 life cycle. Virus Res (2013) 178:517-21.

85. Manaresi E, Bua G, Bonvicini F, Gallinella G. A flow-FISH assay for the quantitative analysis of parvovirus B19 infected cells. J Virol Methods (2015) 223:50-4.

86. Sakata H, Matsubayashi K, Ihara H, Sato S, Kato T, Wakisaka A, Tadokoro K, Yu MY, Baylis SA, Ikeda H, Takamoto S. Impact of chemiluminescent enzyme immunoassay screening for human parvovirus B19 antigen in Japanese blood donors. Transfusion (2013) 53:2556-66.

87. Weseslindtner L, Aberle JH, Hedman L, Hedman K. The Chemokine CXCL-10 Is a Marker of Infection Stage in Individuals With DNAemia Due to Parvovirus B19. J Infect Dis (2017) 215:214-220.

88. Gallinella G, Zuffi E, Gentilomi G, Manaresi E, Venturoli S, Bonvicini F, Cricca M, Zerbini M, Musiani M. Relevance of B19 markers in serum samples for a diagnosis of parvovirus B19correlated diseases. J Med Virol (2003) 71:135-9.

89. Kerr S, O'Keeffe G, Kilty C, Doyle S. Undenatured parvovirus B19 antigens are essential for the accurate detection of parvovirus B19 lgG. J Med Virol (1999) 57:179-85. 
90. Manaresi E, Gallinella G, Zerbini M, Venturoli S, Gentilomi G, Musiani M. IgG immune response to B19 parvovirus VP1 and VP2 linear epitopes by immunoblot assay. J Med Virol (1999) 57:174-8.

91. Musiani M, Manaresi E, Gallinella G, Venturoli S, Zuffi E, Zerbini M. Immunoreactivity against linear epitopes of parvovirus B19 structural proteins. Immunodominance of the amino-terminal half of the unique region of VP1. J Med Virol (2000) 60:347-52.

92. Manaresi E, Zuffi E, Gallinella G, Gentilomi G, Zerbini M, Musiani M. Differential IgM response to conformational and linear epitopes of parvovirus B19 VP1 and VP2 structural proteins. J Med Virol (2001) 64:67-73.

93. Soderlund M, Brown CS, Cohen BJ, Hedman K. Accurate serodiagnosis of B19 parvovirus infections by measurement of IgG avidity. J Infect Dis (1995) 171:710-3.

94. Soderlund M, Brown CS, Spaan WJ, Hedman L, Hedman K. Epitope type-specific IgG responses to capsid proteins VP1 and VP2 of human parvovirus B19. J Infect Dis (1995) 172:1431-6.

95. Yliharsila M, Alaranta S, Lahdenpera S, Lahtinen S, Arku B, Hedman K, Soukka T, Waris M. Array-in-well serodiagnostic assay utilizing upconverting phosphor label technology. J Virol Methods (2015) 222:224-30.

96. Wang Y, Hedman L, Perdomo MF, Elfaitouri A, Bolin-Wiener A, Kumar A, Lappalainen M, Soderlund-Venermo M, Blomberg J, Hedman K. Microsphere-based antibody assays for human parvovirus B19V, CMV and T. gondii. BMC Infect Dis (2016) 16:8.

97. de Ory F, Minguito T, Echevarria JE, Del Mar Mosquera M, Fuertes A. Comparative evaluation of tests for detection of parvovirus B19 IgG and IgM. APMIS (2014) 122:223-9.

98. Mirasoli M, Bonvicini F, Dolci LS, Zangheri M, Gallinella G, Roda A. Portable chemiluminescence multiplex biosensor for quantitative detection of three B19 DNA genotypes. Anal Bioanal Chem (2013) 405:1139-43.

99. Mirasoli M, Bonvicini F, Lovecchio N, Petrucci G, Zangheri M, Calabria D, Costantini F, Roda A, Gallinella G, Caputo D, de Cesare G, Nascetti A. On-chip LAMP-BART reaction for viral DNA real-time bioluminescence detection. Sensors and Actuators B-Chemical (2018) 262:1024-1033.

100. Nguyen Y, Renois F, Leveque N, Giusti D, Picard-Maureau M, Bruneval P, Fornes P, Andreoletti L. Virus detection and semiquantitation in explanted heart tissues of idiopathic dilated cardiomyopathy adult patients by use of PCR coupled with mass spectrometry analysis. J Clin Microbiol (2013) 51:2288-94.

101. Legoff J, Feghoul L, Mercier-Delarue S, Dalle JH, Scieux C, Cherot J, de Fontbrune FS, Baruchel A, Socie G, Simon F. Broad-range PCR-electrospray ionization mass spectrometry for detection and typing of adenovirus and other opportunistic viruses in stem cell transplant patients. J Clin Microbiol (2013) 51:4186-92.

102. Takizawa K, Nakashima T, Mizukami T, Kuramitsu M, Endoh D, Kawauchi S, Sasaki K, Momose H, Kiba Y, Mizutani T, Furuta RA, Yamaguchi K, Hamaguchi I. Degenerate polymerase chain reaction strategy with DNA microarray for detection of multiple and various subtypes of virus during blood screening. Transfusion (2013) 53:2545-55.

103. Leveque N, Legoff J, Mengelle C, Mercier-Delarue S, N'Guyen Y, Renois F, Tissier F, Simon F, Izopet J, Andreoletti L. Virological diagnosis of central nervous system infections by use of PCR coupled with mass spectrometry analysis of cerebrospinal fluid samples. J Clin Microbiol (2014) 52:212-7.

104. Metzgar D, Lovari R, Ray K, Baynes D, Drapp D, Frinder M, Vijesurier R, Stemler M, Ofsaiof R, Carolan H, Welk J, Toleno D, Ranken R, Hall TA, Massire C, Sampath R, Blyn LB, Goveia J, Schneider G. Analytical characterization of an assay designed to detect and identify diverse agents of disseminated viral infection. J Clin Virol (2014) 59:177-83. 
105. Ngoi CN, Siqueira J, Li L, Deng X, Mugo P, Graham SM, Price MA, Sanders EJ, Delwart E. The plasma virome of febrile adult Kenyans shows frequent parvovirus B19 infections and a novel arbovirus (Kadipiro virus). J Gen Virol (2016) 97:3359-3367.

106. Bogdanovic G, Pou C, Barrientos-Somarribas M, Bjerkner A, Honkaniemi E, Allander T, Andersson B, Gustafsson B. Virome characterisation from Guthrie cards in children who later developed acute lymphoblastic leukaemia. Br J Cancer (2016) 115:1008-1014.

107. Pan W, Ngo TTM, Camunas-Soler J, Song CX, Kowarsky M, Blumenfeld YJ, Wong RJ, Shaw GM, Stevenson DK, Quake SR. Simultaneously Monitoring Immune Response and Microbial Infections during Pregnancy through Plasma cfRNA Sequencing. Clin Chem (2017) 63:1695-1704.

108. Somasekar S, Lee D, Rule J, Naccache SN, Stone M, Busch MP, Sanders C, Lee WM, Chiu CY. Viral Surveillance in Serum Samples From Patients With Acute Liver Failure By Metagenomic Next-Generation Sequencing. Clin Infect Dis (2017) 65:1477-1485. 


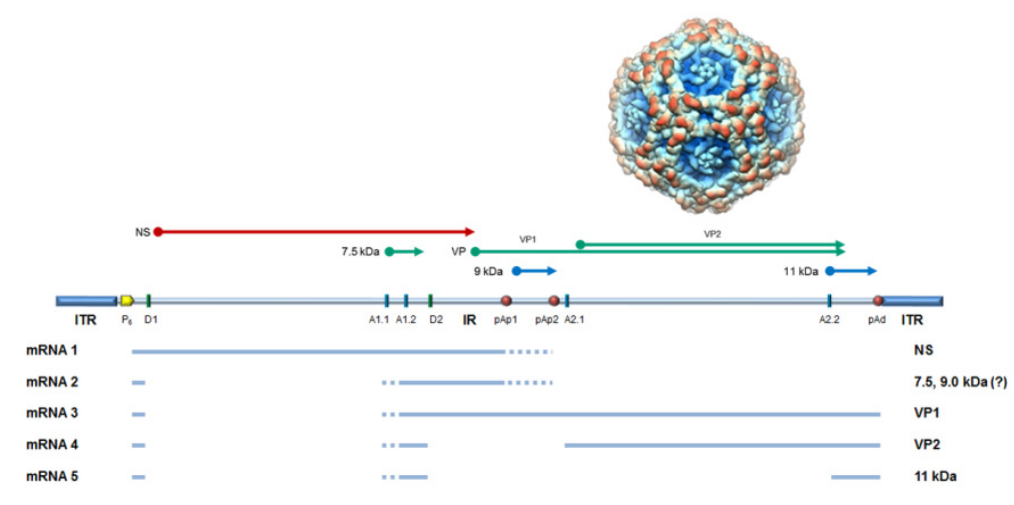

Fig1

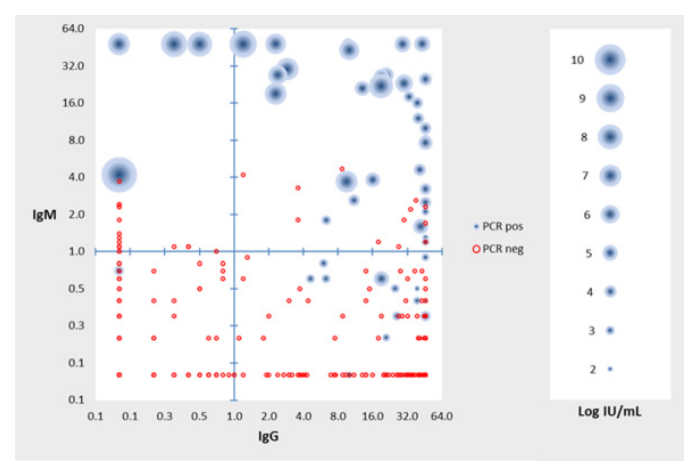

Fig 2 